\title{
Tratamiento de una perforación coronaria durante angioplastía con Stent
}

\author{
Mauricio Aninat H, Humberto Torres H, Sergio Ramos A $^{1}$, Patricia Valenzuela ${ }^{2}$ \\ 1 Tecnólogo Médico. Laboratorio Hemodinamia. Hospital Gustavo Fricke \\ 2Enfermera Universitaria. Laboratorio Hemodinamia. Hospital Gustavo Fricke
}

Laboratorio Hemodinamia y Angiografía. Hospital Dr. G. Fricke. Viña del Mar.

Recibido el 25 de agosto de 2009, aceptado el 30 de octubre de 2009

\section{Introducción}

Una de las complicaciones más temidas de la Angioplastía Coronaria es la perforación o ruptura de la arteria, ya que puede comprometer la vida del paciente. Esta complicación es poco frecuente pero puede presentarse en diversas situaciones dentro de las cuales predominan las técnicas de aterectomías, el uso de algunas guías de angioplastía, las roturas por sobreinflaciones de balones de angioplastía o los implantes de dispositivos de mayor diámetro que la arteria. La perforación coronaria puede revestir diversos grados de gravedad, siendo la rotura coronaria abierta a pericardio la más complicada, ya que produce un hemopericardio de rápida instalación con tamponamiento cardíaco; complicación de alta mortalidad si no se procede de inmediato a sellar la rotura y a drenar el pericardio.

El objetivo de nuestra comunicación es presentar el caso de un paciente que desarrolló esta grave complicación inmediatamente después de la implantación de un stent en el tercio proximal de la coronaria derecha y cuyo tratamiento consistió en sellar la rotura instalando un nuevo stent en el sitio de la perforación.

\section{Caso Clínico}

Paciente varón de 86 años sin antecedentes mórbidos. El 5 de mayo de 2009 debuta con un infarto de pared inferior. No se efectúa terapia de reperfusión pues el paciente consulta tardíamente. Durante los días siguientes presenta episodios de angor de pequeños esfuerzos e incluso de reposo.

El 14 de Mayo de 2009 es sometido a coronariografía, que demuestra lesiones múltiples no significativas del árbol izquierdo y varias lesiones críticas en los tercios inicial y medio de una coronaria derecha dominante (Figura 1).

El flujo arterial coronario se consideró normal (TIMI 3). Dados estos hallazgos y la clínica del paciente, se decide realizar una angioplastía en esta arteria.

El procedimiento se efectuó con un catéter guía AR2 F6. Se utilizó una guía coronaria de 0,014 pulgadas, de extremidad distal flexible, la cual se avanzó a través de las estenosis lográndose una posición distal satisfactoria. Se predilataron las lesiones con un catéter balón de $2,5 \times 14 \mathrm{~mm}$.

Enseguida se avanzó un primer stent de 4,0 x 18mm., el cual se desplegó e impactó en el tercio medio con buen resultado (Figura 2). Se decidió continuar con dilatación de las lesiones más proximales, instalándose a continuación del anterior un stent de $4,0 \times 24 \mathrm{~mm}$ (Figura 3). Por último, se implantó a nivel más proximal, un stent de $4,5 \times 18 \mathrm{~mm}$. Este último, dada la dureza de la lesión, tuvo que desplegarse a alta presión (16 atm.), con lo cual el stent se expandió a $4,85 \mathrm{~mm}$. Al retirar el balón, se observó una perforación de la arteria a nivel medial del stent, con paso masivo de medio de contraste a pericardio (Figura 4). El paciente rápidamente se comprometió hemodinámicamente con hipotensión y evidencias claras de tamponamiento cardíaco.

Correspondencia: Dr. Mauricio Aninat Harnecker

5 Norte 256, Viña del Mar

Teléfono: (032) 297 5411, Fax: (032) 2970058

Correo Eléctronico: maraninat@gmail.com 


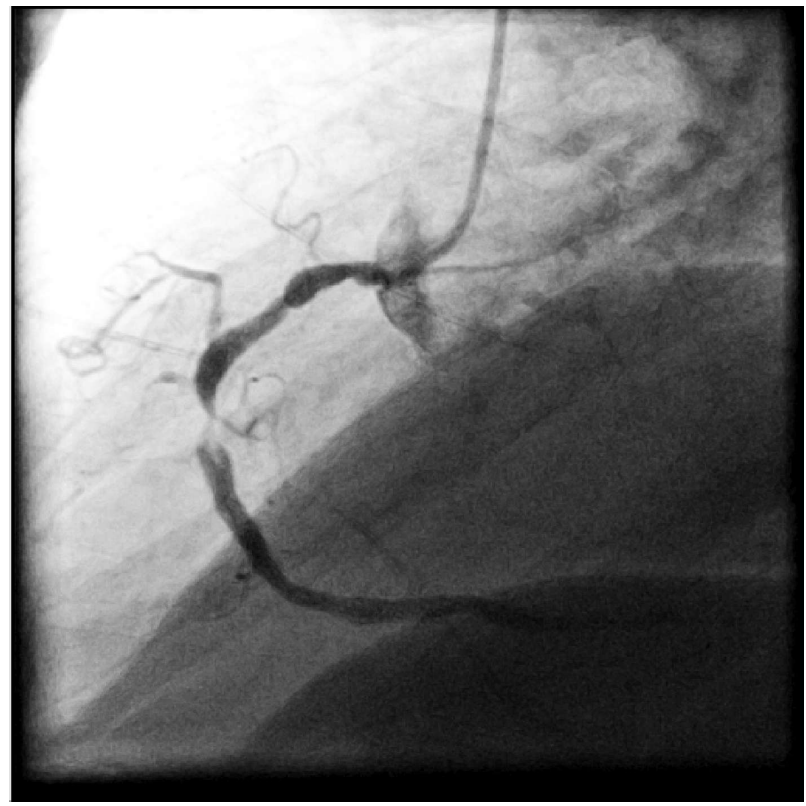

Figura 1: Inyección selectiva en la coronaria derecha que muestra una estenosis crítica medial y varias lesiones proximales.

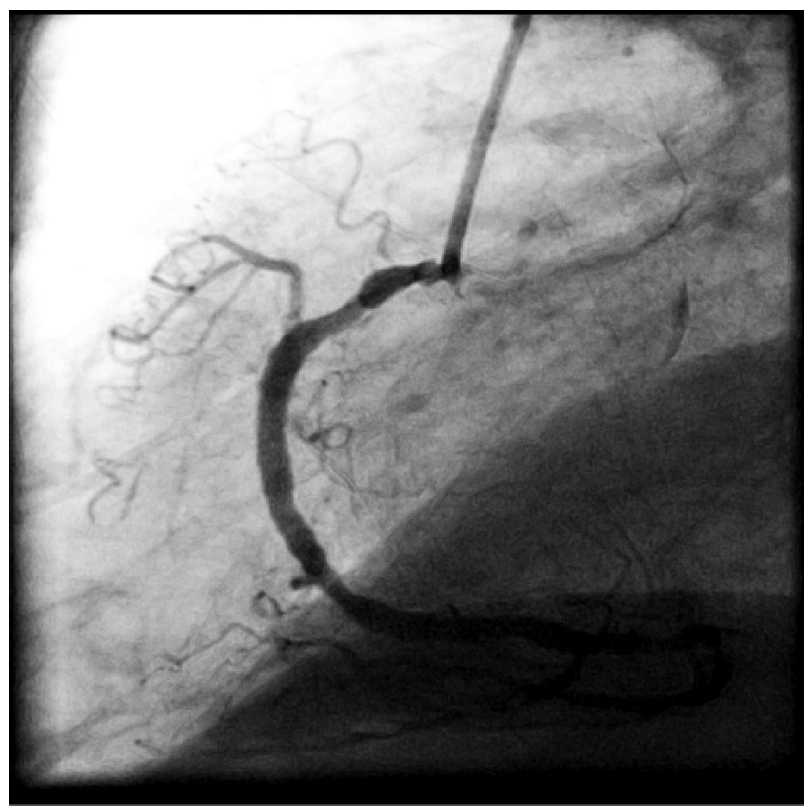

Figura 2: Resultado después del implante del primer stent en el tercio medio de la coronaria derecha. 


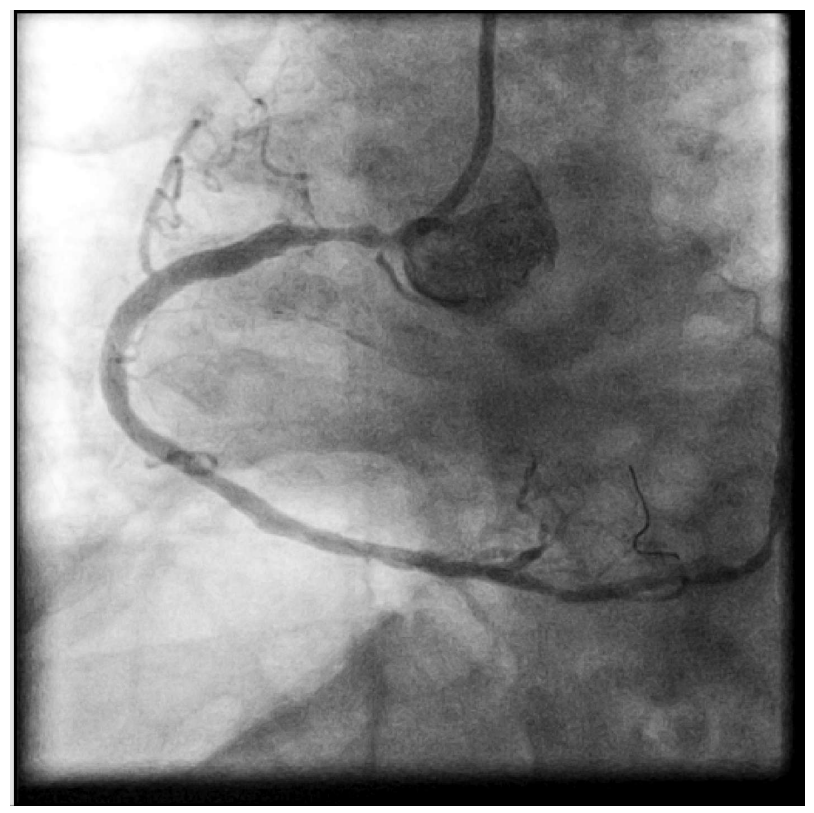

Figura 3: Imagen después del implante del segundo stent en la zona medial proximal de la coronaria derecha.

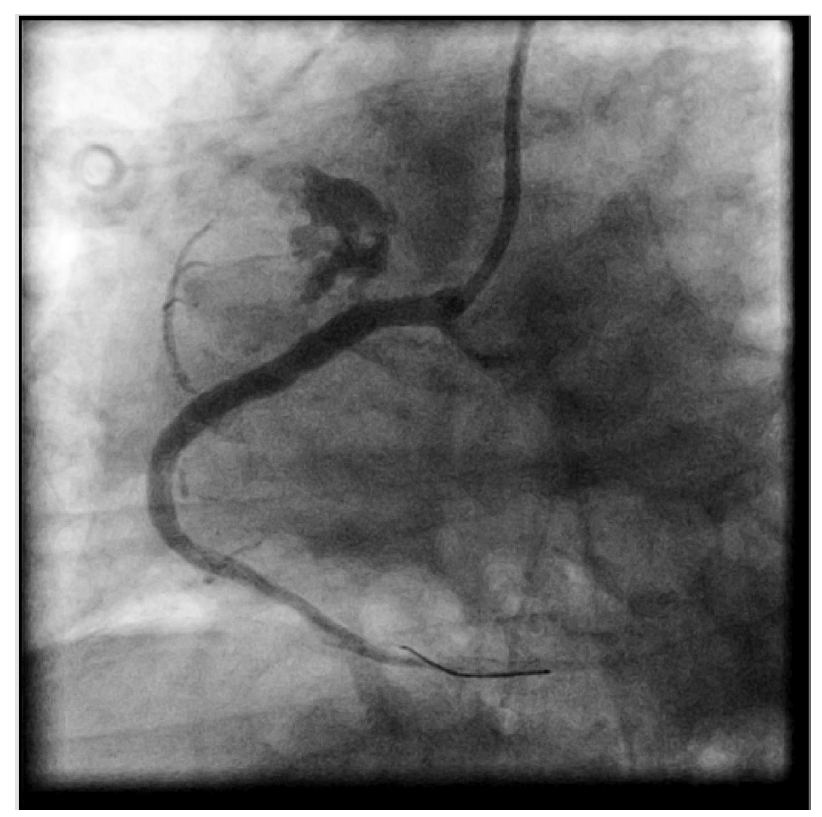

Figura 4: Perforación coronaria en la región media del stent proximal con salida del contraste hacia la cavidad pericárdica.

Ante esta situación catastrófica, se tomó la decisión de avanzar nuevamente el balón del stent expandiéndolo en el sitio de la perforación. Con esta medida se obtuvo una precaria estabilización hemodinámica. Se procedió a administrar protamina para revertir la anticoagulación.

Con el fin de lograr el cierre de la rotura se discutió si usar un stent graft o un stent no recubierto dado que se han reportado casos en que, por aumento de la densidad de la malla o por redistri- 
bución de las placas ateromatosas, se puede lograr el sellado de la arteria. Se decidió usar un stent no recubierto de $4,5 \times 15 \mathrm{~mm}$, el cual se ubicó y se des- plegó en el sitio de la perforación (Fig. 5). Con ello se apreció un excelente resultado con cierre completo de la rotura y buen flujo en la coronaria derecha (Fig. 6).

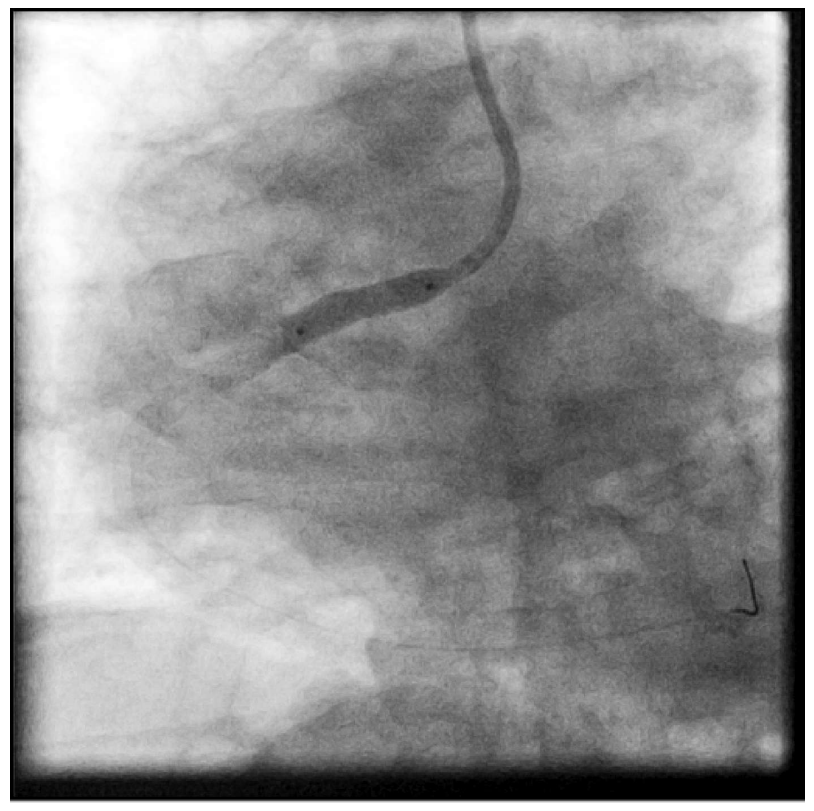

Figura 5: Implante del stent cubriendo la zona de la perforación.

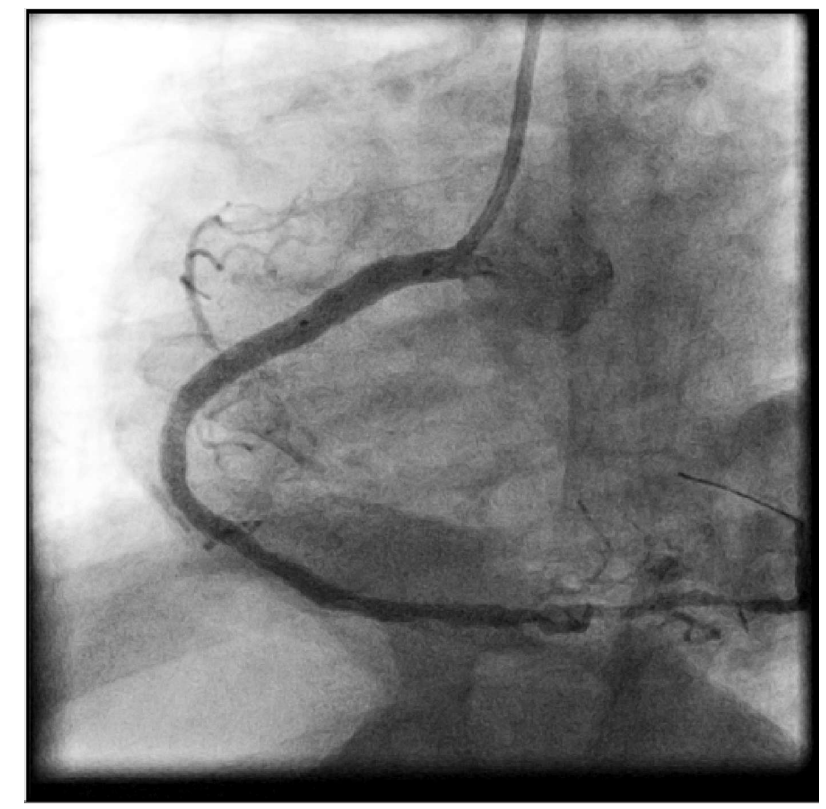

Figura 6: Angiografía de la coronaria derecha que muestra el cierre de la perforación después del implante del stent.

Finalmente, se efectuó punción pericárdica vía subxifoídea obteniéndose $100 \mathrm{cc}$ de sangre, luego de lo cual se logró rápida mejoría hemodinámica. Se instaló un catéter de drenaje pericárdico y el paciente 
fue trasladado a la UTI Cardiovascular en buenas condiciones.

La evolución posterior fue muy satisfactoria. Al día siguiente se retiró el drenaje pericárdico. El paciente fue dado de alta en buenas condiciones.

\section{Discusión}

La perforación coronaria durante una angioplastía es una complicación poco frecuente. Se describe una incidencia entre 0,1 y $3 \%$, pero con la disminución de los procedimientos de aterectomías dicha cifra se acerca a la cifra menor. La gravedad depende de la magnitud de la perforación. Existe una clasificación de la gravedad de las perforaciones en 3 tipos o clases: la perforación Tipo I se define por el desarrollo de un cráter extraluminal de contraste. La perforación Tipo II, por la aparición de una tinción pericárdica o miocárdica sin observarse un jet de extravasación del contraste. La perforación Tipo III se caracteriza por una franca extravasación del medio de contraste a través de ella (mayor de $1 \mathrm{~mm}$.) con salida hacia una cavidad anatómica1. Las perforaciones Tipo I y Tipo II son causadas, habitualmente, por el uso de guías hidrofílicas o por guías de extremos rígidos.

La perforación Tipo III ocurre con procedimientos de aterectomía rotacional o con el implante de stents y se asocia al tratamiento de lesiones calcificadas ${ }^{2}$.

La gravedad en la evolución de la perforación está dada, ya sea por el desarrollo de tamponamiento cardíaco, por la necesidad de efectuar una intervención quirúrgica o por su mortalidad y se acentúa, evidentemente, con la progresión del grado de rotura coronaria. La perforación Tipo I rara vez produce tamponamiento $o$ isquemia y se confunde con disecciones localizadas. La perforación Tipo II constituye una extravasación limitada $y$ se resuelve habitualmente con inflaciones de balones, a veces prolongadas, y tiene una baja incidencia de secuelas. La perforación Tipo III se asocia a una alta incidencia de complicaciones y presenta una elevada mortalidad (sobre 30\%)2,3. El tratamiento en estos casos consiste en sellar la perforación mediante el empleo de un balón inflado en el sitio de la rotura. Se procede a revertir la anticoagulación con protamina. Si la oclusión de la arteria es bien tolerada, se puede proceder a efectuar inflaciones prolongadas con las cuales, ocasionalmente, se podría controlar la perforación. Sin embargo, lo habitual es que se tenga que continuar contemplando otras medidas entre las cuales se propugna la instalación de stents recubiertos con una capa impermeable de politetrafluoroetileno (PTFE) 4 .

De no disponerse de éstos, se puede intentar el implante de un stent desnudo en el sitio de la perforación. El mecanismo de sellado consistiría principalmente en un reacomodo de la lesión ateroesclerótica por desplazamiento de sus capas y el aumento de la densidad de la malla de los stents.

\section{Conclusión}

Se presenta el caso de una de las complicaciones más graves de la Angioplastía con Stent, cual es la perforación coronaria severa con salida masiva de sangre hacia el pericardio y rápida instalación de un colapso hemodinámico por tamponamiento cardíaco. Esta situación fue producida por el implante de un stent en una lesión dura calcificada, que requirió una inflación del stent con alta presión. La complicación fue solucionada satisfactoriamente con el implante de otro stent que logró sellar la rotura y con el drenaje pericárdico percutáneo. Finalmente, se postula una hipótesis sobre el mecanismo que produciría el sellado de la perforación en estos casos.

\section{Referencias}

1. ELLIS SG, AJLUNI S, ARNOLD AZ, POPMA JJ, BITTL $J A$, EIGLER NL, et al. Increased coronary perforation in the new device era. Incidence, classification, management, and outcome. Circulation 1994; 90: 2725-2730.

2. RAMANA RK, ARAB D, JOYAL D, STEEN L, CHO L, LEWIS $B$, et al. Coronary artery perforation during percutaneous coronary intervention: incidence and outcomes in the new interventional era. J Invasive Cardiol 2005; 17: 603-605.
3. JAVAID A, BUCH AN, SATLER LF, KENT KM, SUDDATH WO, LINSAY J, et al. Management and outcomes of coronary artery perforation during percutaneous intervention. Am J Cardiol 2006; 98: 911-914.

4. LY H, AWAIDA JP, LESPERANCE J, BILODEAU L. Angiographic and clinical outcomes of polytetrafluoroethylenecovered stent use in significant coronary perforations. Am J Cardiol 2005; 95: 244-246. 